\title{
Immunohistochemical Analysis of Clinically Transplanted Muscles ${ }^{1}$
}

\author{
Kotaro Yoshimura, M.D.,* Kiyonori Harii, M.D.,* Hirotaka Asato, M.D.,* \\ Kazuki, Ueda, M.D.,† and Atsushi Yamada, M.D.† \\ *Department of Plastic and Reconstructive Surgery, University of Tokyo, Tokyo 113, J apan; †Division of Plastic \\ and Reconstructive Surgery, J ichi Medical College, Tochigi, J apan; and łDepartment of Plastic and \\ Reconstructive Surgery, Tohoku University, Sendai, J apan
}

Submitted for publication February 4, 1998

Backgrounds. Although a number of studies have examined the morphology and function of experimentally transplanted muscles, immunohistochemical evaluation of clinically transplanted muscles has not been reported. The purpose of this study was to examine clinically transplanted muscles at long periods after transplantation with biochemical markers specific for satellite cell activation and muscle regeneration.

Materials and Methods. Nine biopsies of muscles transplanted to the paralyzed face were examined. In five cases, the gracilis muscles were transplanted about 1 year after cross face nerve grafting. The other four cases underwent one-stage latissimus dorsi (LD) muscle transplantation. Twelve to 162 months after transplantation, muscle biopsies were harvested in nine cases. In eight cases, secondary corrections of facial expression including debulking of the grafted muscle were required, while another muscle was transplanted in one case because of the failed first operation. As control, six specimens of normal LDs were examined as well. Monoclonal antibodies were employed to visualize myosin heavy chain (MHC) isoforms (slow, fast, and embryonic) and MyoD protein.

Results. Although one specimen exhibited only small, atrophic fibers indicating failed reinnervation, the remaining eight specimens showed regularly distributed fibers and type grouping indicating successful reinnervation. There was no statistically significant difference in fiber area and lesser diameter between normal LDs and transplanted LDs. However, even in these successfully reinnervated muscles, intermediate and small fibers expressing embryonic MHC and small cells expressing MyoD were observed, suggesting that satellite cells were activated for repair of the adjacent fibers.

Conclusions. Muscle adaptation (presumably to denervation), which is a regenerative change accompanied by activation of satellite cells, was still seen even long peri-

\footnotetext{
${ }^{1}$ The preliminary report of this study was presented at the 7th Annual Meeting of the American Society for Peripheral Nerve, Williamsburg, Virginia, J une 5-7, 1997.
}

ods after transplantation. It is concluded that, in microneurovascular human skeletal muscle transfers, there is a wide variation in the time required for reinnervation of individual muscle fibers, and it may be that human muscle fibers cannot be properly reinnervated after denervation has continued for a certain period such as 12 months. $\odot 1998$ Academic Press

Key Words: transplantation; human; muscle; MyoD; satellite cell; embryonic myosin; muscle adaptation; denervation.

\section{INTRODUCTION}

Although microneurovascular free muscle transplantation has been established as a surgical treatment for peripheral nerve palsies since the 1970s [1, 2], the large deficits in contractile properties of the transplanted muscles still continue to be a problem. A number of factors affecting the function of transplanted muscles such as muscle denervation followed by reinnervation [3, 4], tenotomy [5], and ischemia followed by reperfusion injury [6-9] have been experimentally investigated. A number of modifications and refinements of the techniques including the choice of the donor muscles or the recipient nerve have been re ported [10-14].

A number of studies have examined the morphology and function of experimentally transplanted muscles. However, because of the difficulty of harvesting the clinically transplanted muscle biopsies, there are only a few reports [15] showing the morphology of clinically transplanted muscles, and an immunohistochemical evaluation of clinically transplanted muscles has never been reported. In the present study, we examined nine biopsies of transplanted muscles harvested at the time of the secondary operation. The purpose of this study was to examine immunohistochemically the clinically transplanted muscles at long periods after transplantation using biochemical markers specific for satellite cell activation and muscle regeneration. 
TABLE 1

\section{Summarized Data of Muscle Transplantation Cases (T1-T8)}

\begin{tabular}{|c|c|c|c|c|c|c|c|c|}
\hline Case & $\begin{array}{c}\text { Age } \\
\text { (years) }\end{array}$ & Sex & Operation & Donor & $\begin{array}{l}\text { time at harvest } \\
\text { (months) }\end{array}$ & $\begin{array}{c}\text { Muscle } \\
\text { contraction }\end{array}$ & $\begin{array}{l}\text { Evoked } \\
\text { potential }\end{array}$ & $\begin{array}{c}\text { Voluntary } \\
\text { action potential }\end{array}$ \\
\hline T1 & 26 & Female & CFN & Gracilis & 12 & ++ & - & - \\
\hline T2 & 49 & Male & CFN & Gracilis & 30 & +++ & + & + \\
\hline T3 & 54 & Female & CFN & Gracilis & 162 & +++ & + & + \\
\hline T4 & 38 & Male & CFN & Gracilis & 24 & +++ & + & + \\
\hline T5 & 41 & Female & CFN & Gracilis & 27 & - & - & - \\
\hline T6 & 55 & Male & One-stage & LD & 18 & +++ & + & + \\
\hline T7 & 47 & Female & One-stage & LD & 25 & +++ & + & + \\
\hline Т8 & 53 & Female & One-stage & LD & 12 & +++ & + & + \\
\hline T9 & 52 & Female & One-stage & LD & 17 & +++ & + & + \\
\hline
\end{tabular}

Note CFN, two-stage muscle transplantation following cross facial nerve grafting; one-stage, one-stage muscle transplantation using contralateral facial nerve as a recipient motor source. Clinical muscle contraction was classified from +++ (too strong) to $-($ poor $):+++($ too strong), ++ (strong), + (fair), and - (poor).

\section{MATERIALS AND METHODS}

Biopsies of transplanted muscles. Nine biopsies of muscles transplanted to the paralyzed face were analyzed in this study: five gracilis muscles and four latissimus dorsi muscles. The data of the nine facial palsy cases (T1-T9) are summarized in Table 1 . In cases T1 to $\mathrm{T} 5$, the gracilis muscles were transplanted about 1 year after cross face nerve grafting [11]. The other four cases underwent one-stage latissimus dorsi (LD) muscle transplantation using the contralateral facial nerve as a recipient motor source. Twelve to 162 months (mean, 36.3 months) after transplantation, muscle biopsies were harvested at the time of secondary operation. The secondary corrections of facial expression including debulking of the grafted muscle were required in eight cases (T1-T4, T6-T9) with strong or too strong muscle contraction clinically observed, while another muscle was transplanted in one case (T5) because of the failed reanimation. In seven cases, evoked potential and voluntary action potential were clearly recorded, while neither of them were detected in cases T1 and T5. Our evaluation method of EMG was described previously [13]. As controls, six specimens of normal LD muscles (N1-N6) were examined as well. Each muscle specimen was embedded in O.C.T. compound by freezing in isopentene cooled with liquid nitrogen. Tenmicrometer-thick frozen sections were stained with hematoxylin and eosin (HE) for fiber morphometry.

Immunocytochemistry. Three types of monoclonal antibodies specific for myosin heavy chains (MHCs) were utilized in this study: NCL-MHCf, NCL-MHCs (Novocastra Laboratories, UK), and F 1.652 (Developmental Studies Hybrydoma Bank, IO). NCL-MHCf and $\mathrm{NCL}-\mathrm{MHCs}$, respectively, were raised in mice against native myosin from rabbit psoas and soleus muscles [17]. NCL-MHCf reacts with fast $\mathrm{MHC}$ (both $\mathrm{MHC} 2 \mathrm{a}$ and $\mathrm{MHC} 2 \mathrm{~b}$ ) and, possibly, also with fetal (neonatal) MHCs [18]. NCL-MHCs are specific for slow MHC. The specificity of these antibodies has been described previously $[17,18]$. F 1.652 is derived from hybrydomas prepared from mice immunized with human MHC from human leg muscle at the 15th week of gestation, and reacts with embryonic and fetal (neonatal) MHCs. The preparation of F 1.652 and demonstration of its specificity have been outlined previously $[19,20]$. In this paper, the fibers positive for F 1.652 are referred to as expressing the embryonic MHC, which means both embryonic and fetal (neonatal) MHCs. Additionally, MyoD protein antibody [21] (Novocastra Laboratories) was utilized in this study. The procedures of immunocytochemical staining were performed as previously described [22].

Fiber morphometry. The fiber sizes of all transplanted muscles and normal latissimus dorsi muscles were measured. Five fields of each $\mathrm{HE}$-stained section were selected at random under an optical microscope at a magnification of 40×. Each field included 150 to 350 muscle fibers. Fiber area $\left(\mu \mathrm{m}^{2}\right)$ and lesser diameter $(\mu \mathrm{m})$ were mea- sured following binarization of the images with the image analyser LUZEX-FS (Nireco, Tokyo).

Presentation of data and statistical analysis. Results of fiber morphometry are expressed as the means \pm standard errors of the mean (SEM) of each sample. The averaged values of each sample were compared using the Student t test between normal LD and transplanted muscles. Differences were considered significant for $\mathrm{P}$ values $<0.05$.

\section{RESULTS}

$\mathrm{HE}$ staining. The representative $\mathrm{HE}$ stainings of normal LDs (N1-N6) and transplanted muscles (T1-T9) areillustrated in Fig. 1. One specimen (T5) exhibited only small, atrophic fibers indicating failed reinnervation. The remaining eight specimens showed regularly distributed large fibers packed into bundles with minimal fibrous tissue accumulation, suggesting successful revascularization and reinnervation. Smaller and round fibers, however, were occasionally seen, frequently in groups.

Fiber morphometry. The data of area $\left(\mu \mathrm{m}^{2}\right)$ and lesser diameter $(\mu \mathrm{m})$ of all specimens (N1-N6, T1-T8) are shown in Fig. 2 and Table 2. Some transplanted samples exhibited fibers as large as those of normal samples and there was no statistically significant difference in area and lesser diameter between the transplanted LDs (T6-T9) and normal LDs (N1-N6). Case T5 showed no muscle movement and failed reinnervation, resulting in small fiber size.

Immunohistochemical staining for fast and slow MHCS. Fiber type grouping and regularly distributed fibers were seen in all cases except for case T5, suggesting successful revascularization and reinnervation (Fig. 3). Normal LD muscles showed predominantly fast composition, while transplanted LD muscles, cases T6, T7, T8, and T9, showed slower composition. The analysis of fast and slow MHC composition suggested that there were some fibers expressing both fast and slow MHCs in transplanted muscles.

Immunohistochemical staining for embryonic MHC. Muscle fibers positive for embryonic MHC were seen in all transplanted muscle samples, while 

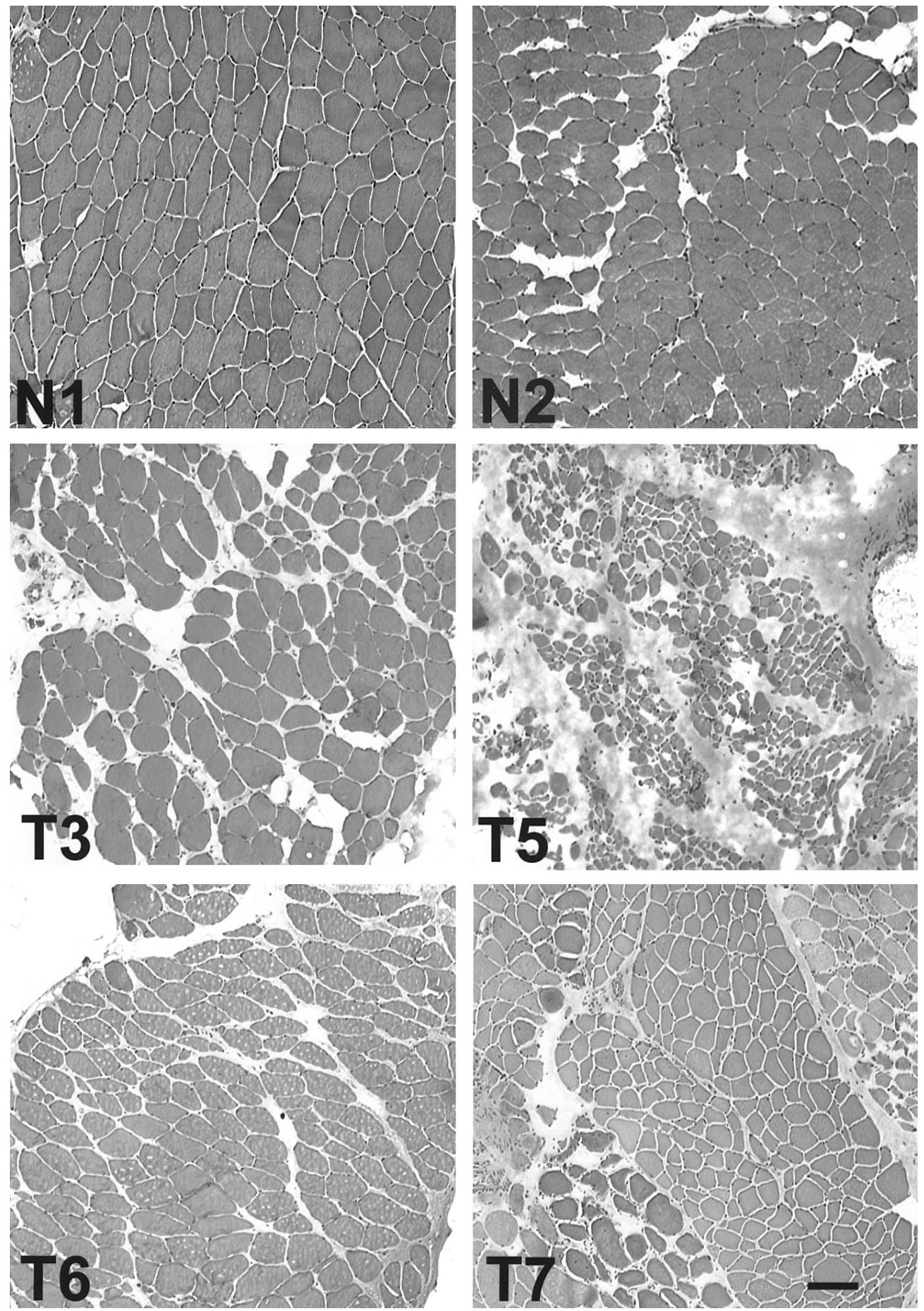

FIG. 1. Hematoxylin and eosin stainings of frozen sections of normal LDs (N1, N2) and transplanted muscles (T3, T5, T6, T7). T5 shows small fibers with accumulated fibrous tissues, suggesting failed reinnervation, probably because of failed revascularization. The others (T3, T6, T7) showed regularly distributed fibers packed into bundles with minimal perimisium, suggesting successful reinnervation. Scale bar, $100 \mu \mathrm{m}$.

they were rarely detected in normal LD muscles (Fig. 4). Additionally, small cells expressing embryonic MHC, suggesting activated satellite cells, were seen in all transplanted samples. In case T6, a muscle spindle, the intrafusal fibers of which were positive for embryonic MHC, was identified.

I mmunohistochemical staining for MyoD protein. Nuclei expressing MyoD protein were also occasionally seen in all transplanted muscle samples, but were scarcely seen in normal LD samples. They were assumed to be the nuclei of newly activated satellite cells (Fig. 5).

\section{DISCUSSION}

Muscle and Nerve Regeneration in Human

Based on experimental results, it had been thought that, with time, reinnervated muscles would finally 


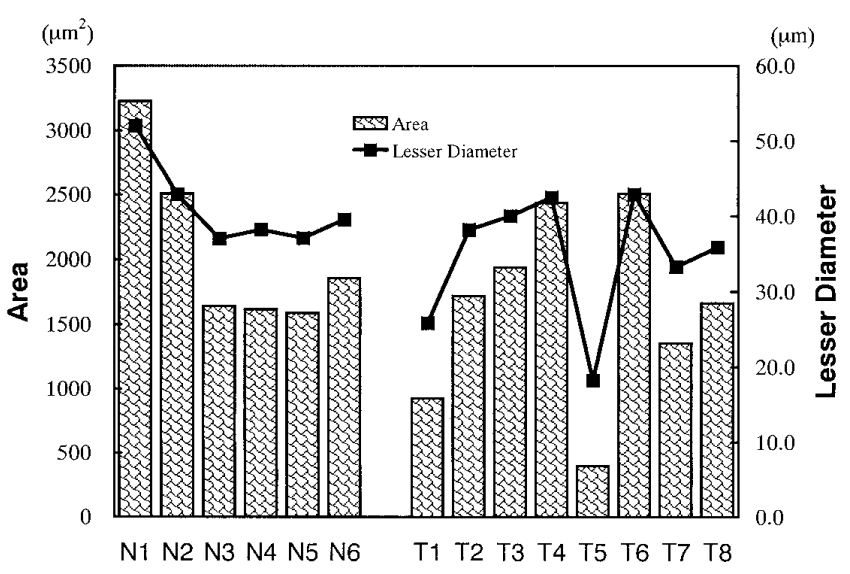

FIG. 2. Averaged fiber area and lesser diameter of normal LDs (N1-N6) and transplanted muscles (T1-T8). T5 is a case of failed reinnervation, probably due to failed revascularization. There is no statistically significant difference in fiber area and lesser diameter between normal LDs and transplanted muscles.

become composed only of reinnervated fibers after nerve repair. It is now known that regeneration of muscle and nerve is several times faster in small animals than in human [23]. Although a number of studies have investigated the morphology and function of experimentally transplanted muscles, differences in the time courses of regeneration between experimental animals and human should always be taken into account when extrapolating such experimental results on restoration of muscle and nerve to the clinical situation. The results of the present study suggest that muscle adaptation is still in progress in clinically transplanted even long periods after transplantation, while the vast majority of muscle fibers are stabilized.

\section{Expression of the Markers for Muscle Regeneration} and Satellite Cell Activation

Expressions of embryonic MHC and MyoD were investigated in the present study as markers for muscle regeneration and satellite cell activation. The different fiber types contain different isoforms of MHC which are primarily responsible for the distinct intrinsic properties of muscle fibers, such as the contraction speed $[24,25]$. MHC expression is developmentally regulated, and four major MHCs are found in developing and adult skeletal muscles: these are the embryonic and neonatal (fetal) isoforms in developing muscles, and the slow and fast isoforms in adult muscles $[26,27]$. Embryonic MHC was found to be expressed in the regenerating process [28] as well as in early muscle development. After activated satellite cells have proliferated, some of them remain as precursor cells for another proliferation in the future and the others begin to differentiate and express embryonic MHC. Embryonic MHC expression is always accompanied by satellite cells activation which can be seen in any kind of muscle adaptation. Indeed, not only after ischemic necrosis but also following denervation or tenotomy, embryonic MHC was expressed, although the amount of expression was much less after denervation than after ischemic necrosis [29].

MyoD is one of the basic helix-loop-helix muscle regulatory factors which regulate skeletal muscle determination and differentiation and can induce nonmyogenic cells to undergo myogenic cell differentiation [21]. MyoD expression was observed in activated satellite cells and lost after they fused into myotubes [30]. The MyoD-positive cells seen in this study were assumed to be newly activated satellite cells, which were not seen in normal adult muscles in which the satellite cells were fully quiescent.

In the present study, in all successfully reinnervated cases, embryonic MHC was expressed in some transplanted muscle fibers, and activated satellite cells expressing embryonic MHC or MyoD were detected. These findings suggest that, unlike normal muscles, all fibers of transplanted human muscles are not stabilized but continue to undergo muscle adaptation even more than 10 years after transplantation.

\section{Muscle Adaptation Seen in Transplanted Muscles}

Although the reason for the muscle adaptation seen in clinically transplanted muscles is not clearly understood, denervation is most likely to be associated with this adaptation. It seemed likely that transplanted muscles which were reinnervated successfully would be stabilized after a long time had elapsed. However, the results of the present study suggest that even more than 10 years after transplantation some fibers of clinically transplanted muscles remain denervated, and in most cases the denervated-like fibers were distributed in groups.

It is suggested that there is a wide variation in the time required for reinnervation of individual muscle fibers, and that human fibers which are kept dener-

\section{TABLE 2}

The Data of Fiber Morphometry of All Normal LDs (N1-N6) and Transplanted Muscles (T1-T8)

\begin{tabular}{lccc}
\hline & $\begin{array}{c}\text { Number of } \\
\text { fibers measured }\end{array}$ & Area $\left(\mu \mathrm{m}^{2}\right)$ & $\begin{array}{c}\text { Lesser diameter } \\
(\mu \mathrm{m})\end{array}$ \\
\hline N1 & 733 & $3225 \pm 62$ & $52.1 \pm 0.5$ \\
N2 & 815 & $2510 \pm 61$ & $42.9 \pm 0.6$ \\
N3 & 1238 & $1641 \pm 26$ & $37.1 \pm 0.4$ \\
N4 & 1146 & $1615 \pm 26$ & $38.2 \pm 0.3$ \\
N5 & 1184 & $1588 \pm 27$ & $37.1 \pm 0.3$ \\
N6 & 1212 & $1855 \pm 30$ & $39.6 \pm 0.3$ \\
T1 & 1825 & $924 \pm 17$ & $25.9 \pm 0.3$ \\
T2 & 1049 & $1720 \pm 44$ & $38.2 \pm 0.5$ \\
T3 & 830 & $1940 \pm 44$ & $40.1 \pm 0.5$ \\
T4 & 783 & $2438 \pm 63$ & $42.5 \pm 0.7$ \\
T5 & 1930 & $397 \pm 8$ & $18.2 \pm 0.2$ \\
T6 & 815 & $2509 \pm 61$ & $42.9 \pm 0.6$ \\
T7 & 1432 & $1352 \pm 27$ & $33.3 \pm 0.4$ \\
T8 & 1193 & $1663 \pm 28$ & $35.9 \pm 0.4$ \\
T9 & 1266 & $944 \pm 26$ & $27.4 \pm 0.3$ \\
\hline
\end{tabular}

Note. Fiber area $\left(\mu \mathrm{m}^{2}\right)$ and lesser diameter $(\mu \mathrm{m})$ were expressed as the means \pm SEM. 

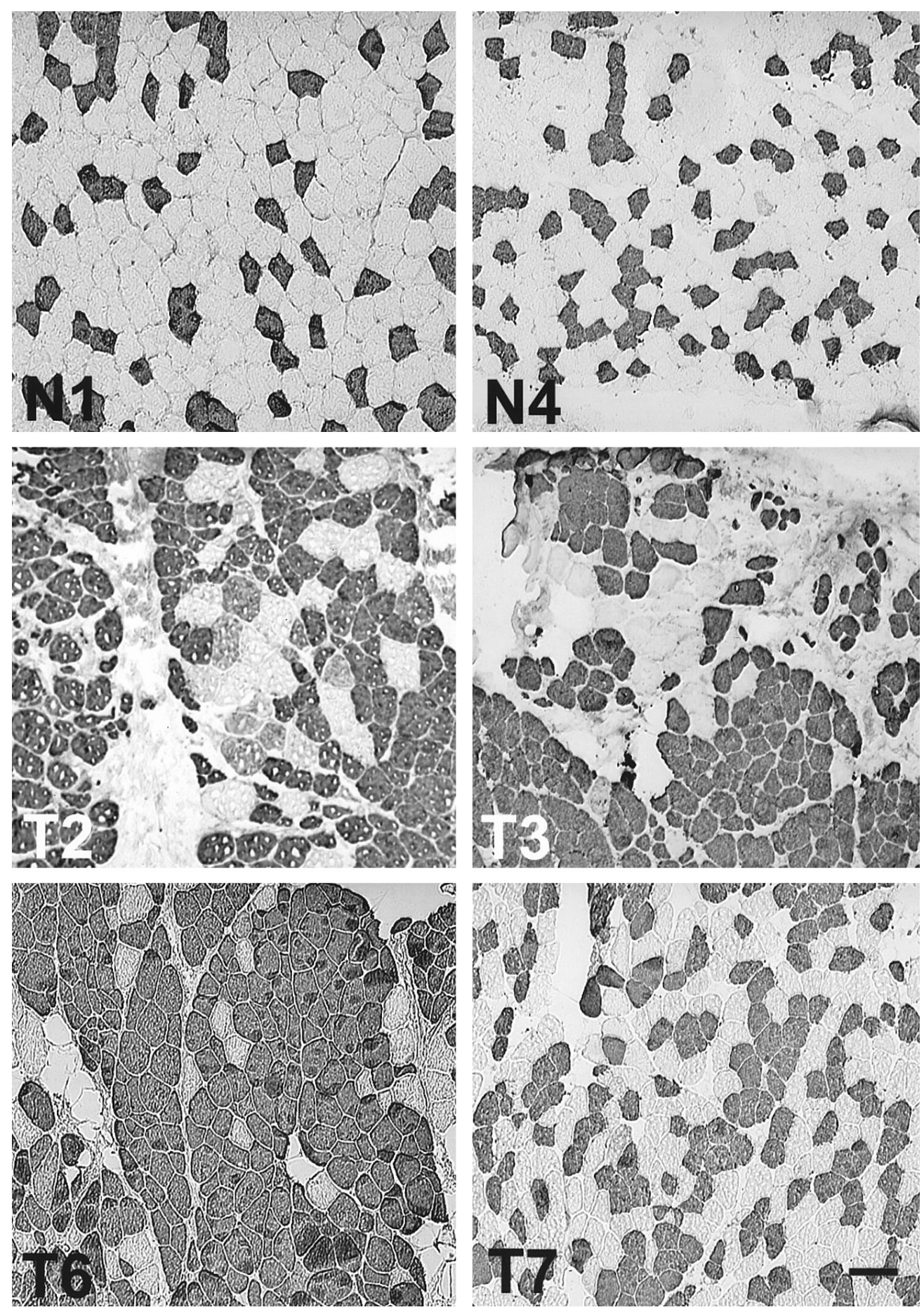

FIG. 3. Immunohistochemical stainings for slow MHC of frozen sections of normal LD (N1) and transplanted muscles (T5, T2, T4, T6, T8). Type grouping and increment of slow fibers are observed in transplanted muscles. Scale bar, $100 \mu \mathrm{m}$.

vated for more than a certain period may lose the ability to become reinnervated. The poor functional recovery after long-term denervation may be derived from atrophied fibers lacking viable satellite cells. Alternatively, it may be due to the deteriorated intramuscular nerve sheaths through which nerve axons would otherwise regenerate [31]. Another possibility is that the recovery is affected by the severing of intramuscular nerve sheaths during surgery. In our series of cases, donor muscles were transected and trimmed before transplantation, and partial muscle transplantation is commonly used for facial reanimation. The authors always confirmed that the trimmed donor muscle contracts properly by electrically stimulating the innervating nerve prior to the elevation of the muscle. However, since it is impossible to ensure all muscle fibers are contracting, the intramuscular nerve sheath to some fibers may have been severed by trimming the donor muscle.

Whereas the reasons for the muscle adaptation and 

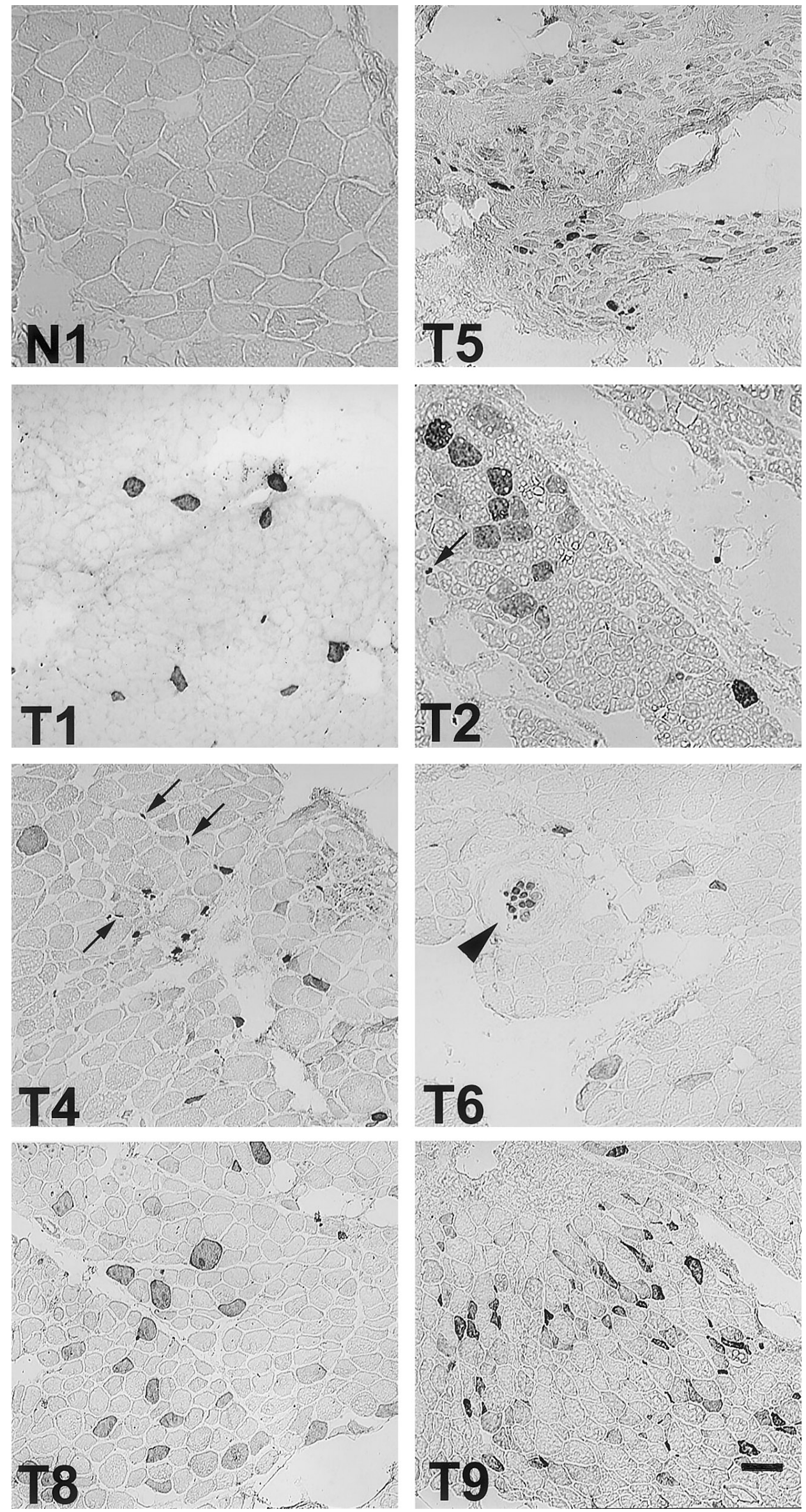

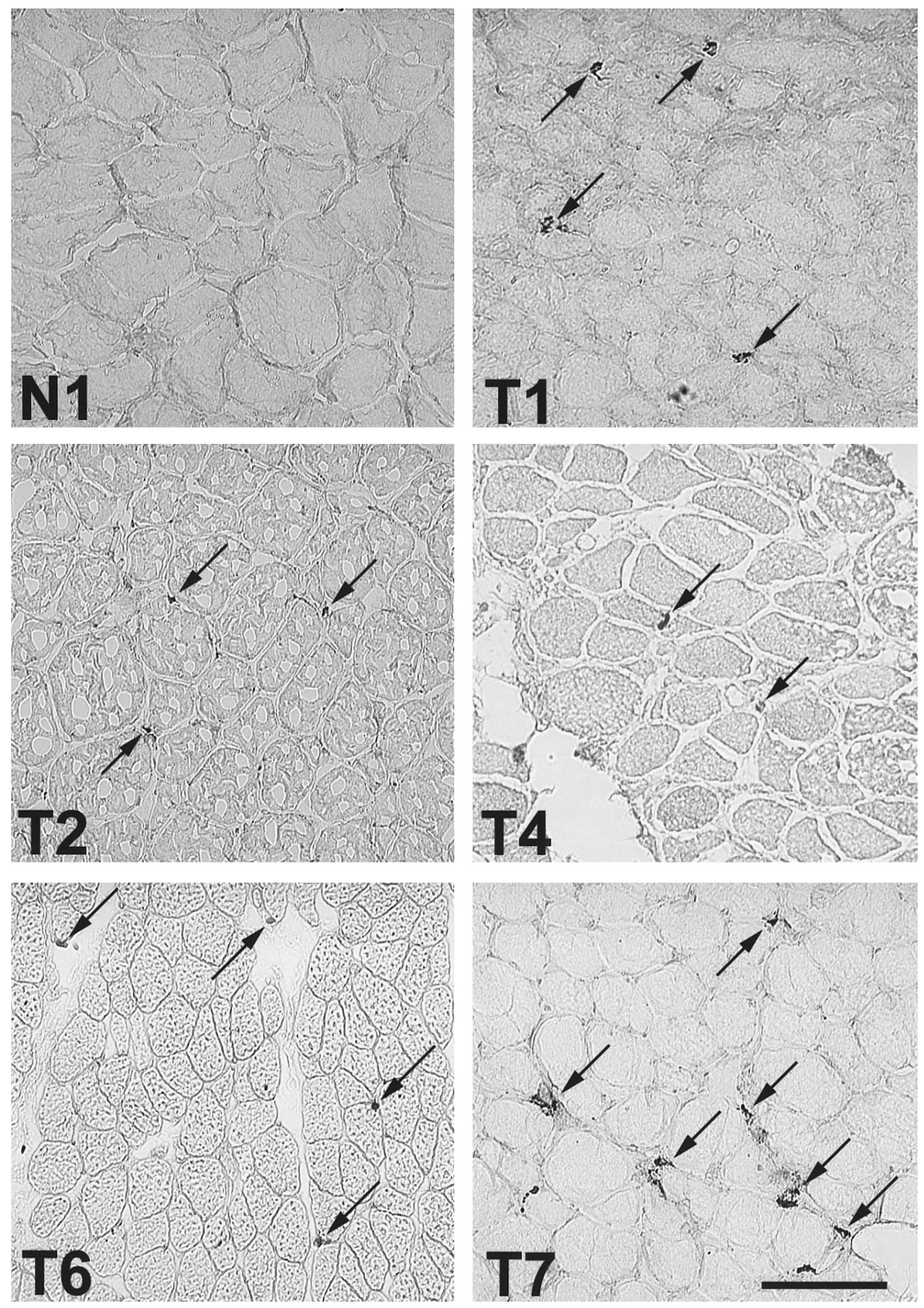

FIG. 5. I mmunohistochemical stainings for MyoD protein of frozen sections of normal LD (N1) and transplanted muscles (T1, T2, T4, T6, T7). In all cases of successful reinnervation, nuclei positive for MyoD are seen (arrows). Scale bar, $100 \mu \mathrm{m}$.

possible partial denervation remain to be elucidated, they are thought to partly contribute tothelarge deficits in the contractile properties of human transplanted muscles. The difficulty in completely reinnervating transplanted muscles may be specific for human because of the very slow regeneration of muscle and nerve.

\section{CONCLUSION}

Muscle adaptation (probably to denervation), which is a regenerative change accompanied by the activation of satellite cells, is still seen even long after the transplantation. In clinically transplanted muscles, some

FIG. 4. Immunohistochemical stainings for embryonic MHC of frozen sections of normal LD (N1) and transplanted muscles (T5, T1, T2, T4, T6, T8, T9). T5, a case of failed reinnervation, showed small fibers expressing embryonic $\mathrm{MHC}$. In all cases of successful reinnervation, fibers positive for embryonic MHC are seen. Small cells (arrows) intensely positive for embryonic MHC are detected as well as large cells, the staining of which varies from faint to intense. Positive intrafusal fibers are also detected in T6 (arrowhead). 
fibers appeared to remain denervated even 2-14 years after transplantation. It is concluded that, in microneurovascular human muscle transfers, there is a wide variation in the time required to obtain reinnervation for individual muscle fibers, and it may be that human muscle fibers cannot be properly reinnervated after denervation has continued for a certain period such as 12 months.

\section{ACKN O WLEDG MENTS}

We express our sincere appreciation to Yuka Kuwahara and Takako Kato for their technical assistance.

\section{REFERENCES}

1. Harii, K., Ohmori, K., and Torii, S. Free gracilis muscle transplantation with microneurovascular anastomoses for the treatment of facial paralysis. Plast. Reconstr. Surg. 57: 133, 1976.

2. I kuta, Y., Kubo, T., and Tsuge, K. Free-muscle transplantation by microsurgical technique to treat severe Volkmann's contracture. Plast. Reconstr. Surg. 58: 407, 1976.

3. Fu, S. Y., and Gordon, T. Contributing factors to poor functional recovery after delayed nerve repair: Prolonged denervation. J . Neurosci. 15: 3886, 1995.

4. Frey, M., Gruber, H., Havel, M., Steiner, E., and Freilinger, G. Experimental free-muscletransplantation with microneurovascular anastomoses. Plast. Reconstr. Surg. 71: 689, 1983.

5. Kadhiresan, V. A., Guelinckx, P. J ., and Faulkner, J . A. Tenotomy and repair of latissimus dorsi muscles in rats: implications for transposed muscle grafts. J . Appl. Physiol. 75: 1294, 1994.

6. Clarke, H. M., and McKee, N. H. Perfusion parameters and pressure at zero flow in canine gracilis muscle flaps. J . Surg. Res. 51: 409, 1991.

7. Quinones-Baldrich, W. J., Chervu, A., Hernandez, J . J ., Colburn, M., and Moore, W. S. Skeletal muscle function after ischemia: "no reflow" versus reperfusion injury. J . Surg. Res. 51: 5, 1991.

8. Fish, J. S., McKee, N. H., Pynn, B. R., Kuzon, W. M., J r., and Plyley, M. J . Isometric contractile function recovery following tourniquet ischemia. J . Surg. Res. 47: 365, 1989.

9. Chervu, A., Moore, W. S., Homsher, E., and Quinones-Baldrich, W. J. Differential recovery of skeletal muscle and peripheral nerve function after ischemia and reperfusion. J . Surg. Res. 47: 12, 1989.

10. Manktelow, R. T., and Zuker, R. M. Muscle transplantation by fascicular territory. Plast. Reconstr. Surg. 73: 751, 1984.

11. Harii, K. Refined microneurovascular free muscle transplantation for reanimation of paralyzed face. Microsurgery 9: 169, 1988.

12. Terzis, J . K. Pectoralis minor: A unique muscle for correction of facial palsy. Plast. Reconstr. Surg. 83: 767, 1989.

13. Doi, K., Sakai, K. R., I hara, K., Abe, Y., Kawai, S., and Kurafuji, $Y$. Reinnervated free muscle transplantation for extremity reconstruction. Plast. Reconstr. Surg. 91: 872, 1993.

14. Ueda, K., Harii, K., and Yamada, A. Free vascularized double muscle transplantation for the treatment of facial paralysis. Plast. Reconstr. Surg. 95: 1288, 1995.
15. Frey, M., Happak, W., Girsch, W., Bittner, R. E., and Gruber, H. Histomorphometric studies in patients with facial palsy treated by functional muscle transplantation: New aspects for the surgical concept. Ann. Plast. Surg. 26: 370, 1991.

16. Ueda, K., Harii, K., and Yamada, A. Electromyographic study of functional recovery of free vascularized muscles grafted to the face. Plast. Reconstr. Surg. 95: 296, 1995.

17. Ecob-Prince, M., Hill, M., and Brown, W. Immunocytochemical demonstration of myosin heavy chain expression in human muscle. J . Neurol. Sci. 91: 71, 1989.

18. Davis, C. E., Harris, J . B., and Nicholson, L. V. Myosin isoform transitions and physiological properties of regenerated and reinnervated soleus muscles of the rat. Neuromusc. Disord. 1: 411, 1991.

19. Silberstein, L., Webster, S. G., Travis, M., and Blau, H. M. Developmental progression of myosin gene expression in cultured muscle cells. Cell 46: 1075, 1986.

20. Webster, C., Silverstein, L., Hays, A. P., and Blau, H. M. Fast muscle fibers are preferentially affected in Duchenne muscular dystrophy. Cell 52: 503, 1988.

21. Tapscott, S. J ., Davis, R. L., Thayer, M. J ., Cheng, P. F., Weintraub, H., and Lassar, A. B. MyoDl: a nuclear phosphorprotein requiring a myc homology region to convert fibroblasts to myoblasts. Science 242: 405, 1988.

22. Yoshimura, K., Kuzon, W. M., and Harii, K. Myosin heavy chain expression in skeletal muscle autografts under neural or aneural conditions. J . Surg. Res. 75, in press.

23. Carlson, B. M., and Faulkner, J . A. The regeneration of skeletal muscle fibers following injury: A review. Med. Sci. Sports Exercise 15: 187, 1983.

24. Reiser, P. J ., Moss, R. L., Giulian, G. G., and Greaser, M. L. Shortening velocity in single fibers from adult rabbit soleus muscles is correlated with myosin heavy chain composition. J . Biol. Chem. 260: 9077, 1985.

25. Sweeney, H. L., Kushmerick, M. J ., Mabuchi, K., Sreter, F. A., and Gergely, J. Myosin alkali light chain and heavy chain variations correlate with altered shortening velocity of isolated skeletal muscle fibers. J . Biol. Chem. 263: 9034, 1988.

26. Whalen, R. G., Sell, S. M., Butler-Browne, G. S., Schwartz, K., Bouveret, P., and Pinset-Harstom, I. Three myosin heavy-chain isozymes appear sequentially in rat muscle development. Nature 292: 805, 1981.

27. Bandman, E. Continued expression of neonatal myosin heavy chain in adult dystrophic skeletal muscle. Science 227: 780, 1985.

28. Sartore, S., Gorza, L., and Schiaffino, S. Fetal myosin heavy chains in regenerating muscle. Nature 298: 294, 1982.

29. Schiaffino, S., Gorza, L., Pitton, G., Saggin, L., Ausoni, S., Sartore, S., and Lomo, T. Embryonic and neonatal myosin heavy chain in denervated and paralyzed rat skeletal muscle. Dev. Biol. 127: 1, 1988.

30. Koishi, K., Zhang, M., McLennan, I. S., and Harris, A. J . MyoD protein accumulates in satellite cells and is neurally regulated in regenerating myotubes and skeletal muscle fibers. Dev. Dyn. 202: 244, 1995.

31. Fu, S. Y., and Gordon, T. Contributing factors to poor functional recovery after delayed nerve repair: Prolonged denervation. J . Neurosci. 15: 3886, 1995. 